\title{
Night Sweats: A Systematic Review of the Literature
}

\author{
James W. Mold, MD, MPH, Barbara J. Holtzclaw, RN, PhD, FAAN, \\ and Laine McCarthy, MLIS
}

Background: Much of primary care involves helping patients manage symptoms. Nighttime sweating is a symptom linked to menopause, malignancies, autoimmune diseases, and infections. However, in primary care settings, night sweats are commonly reported by persons without these conditions.

Methods: We conducted a literature review, focusing on questions about definition, mechanisms, incidence/prevalence, measurement, clinical causes, evaluation, treatment, and prognosis. We limited our search to English language studies of adult humans published since 1966. Because studies of estrogen and androgen deficiency states had been reviewed by others, we excluded them. Search criteria were developed for each question. Publications meeting criteria were reviewed by the first 2 authors and consensus was reached through discussion.

Results: Prevalence estimates ranged from $10 \%$ among older primary care patients to $60 \%$ among women on an obstetrics inpatient unit. Life expectancy of primary care patients reporting night sweats did not appear to be reduced. Although many clinical causes have been suggested, most are not well supported. Algorithmic approaches to evaluation are not evidence-based. Alpha adrenergic blockers may reduce night sweats in patients taking serotonin reuptake inhibitors. Thalidomide and thioridazine may benefit some terminal cancer patients with night sweats.

Conclusions: The symptom, night sweats, appears to be nonspecific. Many questions about causation, evaluation, and management remain unanswered. (J Am Board Fam Med 2012;25:878-893.)

Keywords: Diaphoresis, Night Sweats, Symptom, Systematic Review, Thermoregulation

Bothersome symptoms, one fourth of which are chronic or recurrent, account for $>50 \%$ of outpatient encounters. ${ }^{1}$ At least one third to one half of these symptoms are never fully explained and others are particularly difficult to interpret. Included among these is the symptom, night sweats.

Patients' concerns about night sweats are fairly common. Ely ${ }^{2}$ identified questions about the cause of night sweats as among the most frequent questions posed by patients for which he could find no evidence-based answer. In an effort to clarify what is currently known about night sweats, the authors

This article was externally peer reviewed.

Submitted 22 February 2012; revised 25 May 2012; accepted 5 June 2012.

From the College of Medicine, University of Oklahoma Health Sciences Center, Oklahoma City, OK.

Funding: none.

Conflict of interest: none declared.

Corresponding author: James W. Mold, MD, MPH, College of Medicine, University of Oklahoma Health Sciences Center, $900 \mathrm{NE} 10^{\text {th }}$ Street, Oklahoma City, OK 73104 (E-mail: James-mold@ouhsc.edu.). undertook a systematic review of the medical literature.

\section{Methods}

We searched the Medline database (Ovid Technologies) from 1966 through 2011 for all articles containing the Medical Subject Heading sweating combined with the text phrase night, which yielded 84 citations before applying the inclusion and exclusion criteria. We also searched using the text phrases night sweats and nocturnal diaphoresis. Text word searching was used because there are no Medical Subject Heading terms specifically addressing night sweats or nocturnal diaphoresis. We then limited our retrieval to English language and to studies categorized as adult and human, alone and in combination with category-specific terms (eg, specific diseases and conditions). We then excluded articles related to estrogen, menopause, perimenopause, and testosterone deficiency. This yielded 509 distinct citations. Reference lists from those publications provided additional citations. 
We also looked at the references provided in the section on night sweats authored by Smetana in Up-to-Date Version 19.6 (Up-To-Date, Inc), an electronic medical resource for clinicians and $\mathrm{pa}-$ tients. $^{3}$

To focus our search for information, we developed a set of 8 questions we believed to be of importance to clinicians and patients trying to understand and manage this symptom: (1) How have night sweats been defined? (2) What mechanisms could be responsible for night sweats? (3) What incidence, prevalence, and epidemiologic variables associated with night sweats are reported in population-based studies? (4) To what extent are subjective reports of night sweats associated with objectively measured night sweats, and how can discrepancies be explained? (5) What clinical conditions have been associated with night sweats? (6) How should patients bothered by night sweats be evaluated? (7) Aside from treatment of the underlying cause, how can night sweats be reduced/ treated? (8) Are night sweats associated with adverse health outcomes?

More specific inclusion and exclusion criteria were then developed for several of the 8 focus questions. For question 3, we limited our review to studies that described a population defined by site rather than medical condition and that assessed all, or a random sample, of members. For factors associated with night sweats, studies were included if there was a comparison group and the researchers used inferential statistics to compare the groups on variables associated with night sweats.

For question 5, we made a list of all the proposed clinical causes of night sweats found in review articles, online summaries, and book chapters. For each proposed condition we conducted searches of the English language literature using the name of the condition plus "night sweats." We then only included cohort studies with a control group, case series of $\geq 10$ individuals, or smaller case series when there was specific information about whether the night sweats resolved with treatment of the underlying condition. A number of studies involving patients with neoplasias were excluded because they grouped "B-type symptoms" (fever, night sweats, and weight loss) together.

The first 2 authors independently reviewed all relevant articles and then met to review and resolve any disagreements regarding their inclusion in the review. Finally, all authors met and reached consensus on final text and tabular content.

\section{Results}

\section{How Have Night Sweats Been Defined?}

The published definitions that we were able to locate are shown in Table 1. For the most part, definitions for night sweats appear to have been developed independently by these authors or passed down by word of mouth since they were rarely referenced. They vary primarily by their severity requirements with some authors proposing

Table 1. Published Definitions of Night Sweats

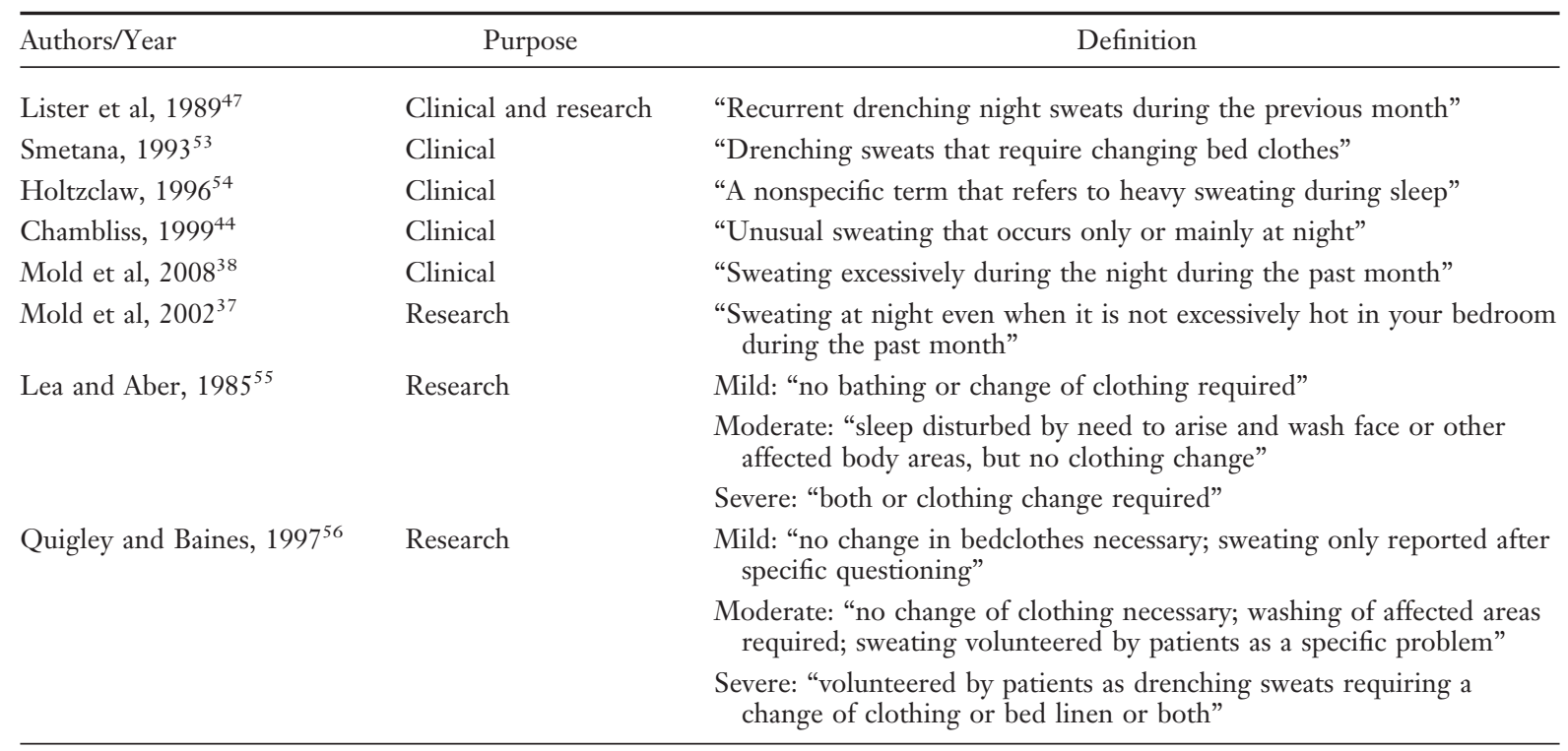


subclassifying night sweats into mild, moderate, and severe. Some definitions specify a time interval, but none includes frequency criteria. Some require the absence of excessive daytime sweating, whereas one restricts nighttime sweating to sweating that occurs only during sleep. Patterns and locations of sweating (palmar/plantar, axillary, facial, truncal, diffuse, etc) are not specified. Most definitions exclude environmental factors such as room temperature or humidity. No published definition requires confirmation by an external observer or an objective test. None of the definitions requires that the symptom be bothersome to the individual or to others.

\section{What Mechanisms Could Be Responsible for Night Sweats?}

Regulation of sweating is complex, involving both thermoregulatory and nonthermoregulatory mechanisms. Despite a very large body of literature on physiological mechanisms related to sweating in humans, ${ }^{4-6}$ we found only a few studies examining mechanisms associated specifically with night sweats. ${ }^{7-9}$ Sweating helps to reduce core body temperature when it rises above certain limits or thresholds, called the thermoneutral zone (TNZ). ${ }^{10}$ Thermoregulatory defenses such as sweating are stimulated when threshold levels in core body temperatures trigger a hypothalamic response. ${ }^{11}$ This can happen because of environmental heat exposure or decreased heat dissipation (eg, excessive clothing or bed coverings) or as a result of increased heat production (eg, excessive muscular activity). Release of inflammatory mediators during infections, autoimmune diseases, and malignancies can temporarily raise the TNZ, inducing chills and shivering that causes core body temperature to rise. Sweating occurs when the levels of these mediators and the TNZ return to normal. ${ }^{12}$ Diurnal patterns of temperature elevation have been explained by nocturnal rise and fall of viral loads or bacteremic showers and periodic increases in interleukins (IL-1 $\alpha$, IL-2, IL-4, IL-6) and tumor necrosis factor but no research was found to support this as a common mechanism responsible for night sweats.

Circadian variations influence sweating and other thermoregulatory responses during exercise $\mathrm{e}^{13,14}$ and throughout the night with sweat thresholds lower in the morning than at any other time of day. ${ }^{15}$ Physical conditioning appears to reduce postexercise sweating thresholds, so sweating is promoted at somewhat lower temperatures than expected. ${ }^{16}$ Whether this might put conditioned athletes at risk for night sweats has not been postulated in the literature; however, Kreider et al listed night sweats as one of the major symptoms of sports overtraining by prevalence in the literature. ${ }^{17}$ Empirical evidence shows that hypothalamic adjustments in thermoregulation occurs after 8 to 14 days of exercise in a hot environment. ${ }^{18}$

Acute and chronic anxiety states and physical conditioning can increase the responsiveness of sweat glands. ${ }^{5,19-21}$ Conditions that reduce the range (difference between lower and upper limits) of the TNZ can result in the need for more frequent adjustments and therefore more frequent sweating. This is believed by some to be a mechanism contributing to sweating during menopausal hot flashes. ${ }^{22}$

Body temperature tends to fall slightly in the evening, and lower core temperature appears to facilitate sleep. ${ }^{23}$ Depression is associated with loss of the usual reduction in core temperature that occurs in the evening and nighttime. ${ }^{24-30}$ One study found that depressed patients sweat more at night than control subjects but only during the 20 minutes just before the onset of rapid eye movement sleep. ${ }^{31}$ Sweating increases with increasing depths of nonrapid eye movement sleep. It is reduced during rapid eye movement sleep in the absence of emotionally charged dreams despite increases in brain glucose metabolism, increased temperature in many parts of the brain, increase skin sympathetic activity, and increased heart rate. ${ }^{31-36}$

Nonthermoregulatory regulation of sweating could also be involved in some people who report night sweats. Sweating can result from a wide variety of medications that affect the sympathetic nervous system, the thermoregulatory center, or the sweat glands; and from sleep stage disturbances, autonomic nervous system disorders, medullary and spinal cord abnormalities, reductions in serum osmolality or abnormalities of osmoreceptor function, hypercapnia, and direct sweat gland stimulation by pressure, heat, trauma, or toxins. ${ }^{3}$ However, we could find no published studies beyond letters to the editor or single case studies linking any of these potential mechanisms to night sweats.

Some people who report night sweats may simply be more aware or concerned about nighttime sweating because they are awake for other reasons. 
Table 2. Incidence and Prevalence of Night Sweats in Various Populations

\begin{tabular}{|c|c|c|c|}
\hline Authors/Year & Population (Country) & $\mathrm{N}$ & Prevalence and Incidence Estimates \\
\hline Lea and Aber, $1985^{55}$ & Hospital inpatients (US) & 174 & $\begin{array}{l}\text { Prevalence: medicine: } 33 \% \\
\text { Surgery: } 33 \% \\
\text { Obstetrics: } 60 \% \\
\text { Gynecology: } 27 \%\end{array}$ \\
\hline Reynolds, $1989^{57}$ & $\begin{array}{l}\text { Consecutive patients seen in a gastroenterology } \\
\text { practice* }^{*}\end{array}$ & 200 & Prevalence: $41 \%$ \\
\hline Quigley and Baines, $1997^{56}$ & $\begin{array}{l}\text { Hospice inpatients patients, consecutive } \\
\text { admissions (UK) }\end{array}$ & 100 & $\begin{array}{l}\text { Prevalence: } 16 \% \text { overall } \\
12 \% \text { soaked bed clothes }\end{array}$ \\
\hline Mold et al, $2002^{37}$ & Primary care patients, consecutive visits (US) & 2267 & $\begin{array}{l}\text { Prevalence: all: } 41 \% \\
\text { Nighttime only: } 23 \% \\
4-7 \text { nights per week: } 16 \%\end{array}$ \\
\hline Mold et al, $2004^{40}$ & $\begin{array}{l}\text { Primary care patients }>65 \text { years of age, recruited } \\
\text { from billing records (US) }\end{array}$ & 795 & Prevalence: $10 \%$ \\
\hline Mold et al, $2006^{39}$ & Primary care patients, consecutive visits (US) & 363 & $\begin{array}{l}\text { Prevalence: } 34 \% \\
17 \% \text { soaked bed clothes }\end{array}$ \\
\hline Mold et al, $2008^{38}$ & $\begin{array}{l}\text { Consecutive patients undergoing } \\
\text { polysomnography (US) }\end{array}$ & 282 & Prevalence: $28 \%$ \\
\hline Mold and Lawler, $2010^{46}$ & $\begin{array}{l}\text { Consecutive patients }>65 \text { years of age enrolled in } \\
\text { a primary care geriatric continuity clinic }(\mathrm{US})^{*}\end{array}$ & 682 & Prevalence: $14 \%$ past year \\
\hline Mold and Lawler, $2010^{46}$ & $\begin{array}{l}\text { Primary care patients }>65 \text { years of age, recruited } \\
\text { from billing records (US) }\end{array}$ & 795 & Incidence (annual): $5 \%$ \\
\hline Suwanprathes et al, $2010^{58}$ & Random population sample, (Bangkok, Thailand)* & 4680 & Prevalence: $4.4 \%$ \\
\hline
\end{tabular}

*Night sweats definition not provided in report.

Some individuals may be less tolerant of either sweat or its cooling effect or anxious about symptoms, like night sweats, that might indicate illness. One study, which reported an association between night sweats and sleep problems in men, supports this hypothesis. ${ }^{37}$ Two studies reporting associations between subjective night sweats and awakening due to pain or a bitter taste also support it. ${ }^{38,39}$ In the 2004 study by Mold et al, ${ }^{40}$ individuals who reported night sweats were also more likely to report many other symptoms about which they were queried. In a primary care population in South Korea in which the researchers used methods identical to those used in the 2002 study by Mold et al, researchers found a prevalence of only $21 \%$ compared with $41 \%$ in the US study, suggesting that either night sweats are less common in South Korea or that South Koreans are less likely to notice or be willing to report this symptom. ${ }^{41}$

\section{What Incidence, Prevalence, and Epidemiologic Variables Associated with Night Sweats Are Reported in Population-based Studies?}

In all the studies reviewed, individuals were asked specifically about night sweats and did not sponta- neously report the symptom. In Table 2 we have listed the incidence and prevalence rates of night sweats reported in these populations. (The definitions used are listed in Table 1.) Table 3 lists variables found to be associated with the subjective report of night sweats in these population-based studies.

Prevalence estimates have varied from $10 \%$ in older patients recruited from primary care billing records to $41 \%$ in consecutive patients being seen in primary care or gastroenterology outpatient settings. The only incidence estimate $(5 \%)$ was in the geriatric cohort recruited from primary care billing records.

\section{To What Extent Are Subjective Reports of Night Sweats Associated with Objectively Measured Sweating?}

We found no published studies addressing this question.

\section{What Clinical Conditions Have Been Associated with Night Sweats?}

Night sweats have been associated with a long list of clinical conditions. ${ }^{42-45}$ Table 4 lists all the 
Table 3. Variables Associated with Night Sweats in Epidemiological Studies

\begin{tabular}{|c|c|c|c|}
\hline Authors/Year & Study Design & Analytic Method & Factors Associated with Night Sweats \\
\hline Lea and Aber, $1985^{55}$ & Cross-sectional & $x^{2}$ & Aspirin or acetaminophen use $(P<0.05)$ \\
\hline \multirow[t]{4}{*}{ Mold et al, $2002^{37}$} & \multirow[t]{4}{*}{ Cross-sectional } & \multirow[t]{4}{*}{ Logistic regression } & All: panic attacks $(\mathrm{OR}, 4.8 ; 1.7-13.6)^{*}$ \\
\hline & & & Men: sleep problems (OR, 2.5; 1.7-3.8) \\
\hline & & & Women: hot flashes (OR, 3.4; 1.1-10.0) \\
\hline & & & Panic attacks (OR, 4.5; 1.2-16.7) \\
\hline \multirow[t]{9}{*}{ Mold et al, $2004^{40}$} & \multirow[t]{9}{*}{ Cross-sectional } & \multirow[t]{5}{*}{ Logistic regression } & Age (OR, 0.94/year; 0.89-0.98) \\
\hline & & & Fever (OR, 12.6; 6.6-24.1) \\
\hline & & & Numbness hands/feet (OR, 3.3; 1.9-5.8) \\
\hline & & & Muscle cramps (OR, 2.8; 1.5-5.2) \\
\hline & & & Impaired vision-subjective (OR, 2.5; 1.4-4.3) \\
\hline & & \multirow[t]{4}{*}{ Cluster analysis } & Impaired hearing-subjective (OR, $1.8 ; 1.0-3.3)$ \\
\hline & & & Symptoms of anxiety and stress $(P=0.001)$ \\
\hline & & & Symptoms of dysphoria $(P=0.001)$ \\
\hline & & & Pain and discomfort $(P<0.0001)$ \\
\hline \multirow[t]{4}{*}{ Mold et al, $2006^{39}$} & \multirow[t]{4}{*}{ Cross-sectional } & \multirow[t]{4}{*}{ Logistic regression } & Daytime tiredness (OR, 2.0; 1.1-3.5) \\
\hline & & & Waking with bitter taste (OR, 1.9; 1.2-3.2) \\
\hline & & & Awakening with pain $(\mathrm{OR}, 1.9 ; 1.2-3.0)$ \\
\hline & & & Legs jerk in sleep $(\mathrm{OR}, 1.8 ; 1.1-3.0)$ \\
\hline \multirow[t]{3}{*}{ Mold et al, $2008^{38}$} & \multirow[t]{3}{*}{ Cross-sectional } & \multirow[t]{3}{*}{ Logistic regression } & Trouble breathing at night (OR, 2.8; 1.5-5.2) \\
\hline & & & Awakened by aches and pains (OR, 3.2 (1.8-5.8) \\
\hline & & & Epworth Sleepiness Scale (OR, $1.1(1-1,1)$ \\
\hline
\end{tabular}

*ORs with $95 \%$ CIs.

CI, confidence interval; OR, odds ratio.

conditions for which we found data meeting our inclusion and exclusion criteria. In Table 5, we have listed the prevalence of night sweats among individuals in all the larger case series $(\geq 10$ cases) believing that comparing this information with the prevalence estimates from Table 2 might provide additional information regarding the strength of the associations between night sweats and specific medical conditions. Some series therefore appear in both Table 4 and Table 5 .

Although mentioned in a least one review or book chapter, we could find no published studies meeting our inclusion criteria that support an association between night sweats and any of the following conditions: mixed connective tissue disease, polymyalgia rheumatica, polymyositis, dermatomyositis, Schnitzler syndrome, scleroderma, systemic lupus erythematosus, congestive heart failure, carcinoid syndrome, insulinoma, pheochromocytoma, brucellosis, dental abscess, lung abscess, fungal pneumonia, sinusitis, Castleman disease, oat cell carcinoma of the lung, renal cell carcinoma, reticulum cell carcinoma, splenic hamartoma, autonomic neuropathy, autonomic dysreflexia, dysautonomia, anterior hypothalamic lesions, dorsolateral midbrain lesions, HinesBannick syndrome, multiple sclerosis, myasthenia gravis, Parkinson disease, pontine lesions, spinal cord transection, syringomyelia, bulimia, rickets, scurvy, alcoholism, anxiety, situational stress, night terrors, alcohol or benzodiazepine withdrawal, narcotic withdrawal, eosinophilic pneumonia, gout, immersion foot syndrome, Pink disease, or uremia. We also found no qualifying evidence for an association between night sweats and the following medications: anticholinergics, antipyretics, antitussives, antispasmodics, acetaminophen, $\beta$-blockers, decongestants, insulin, meperidine, nonsteroidal anti-inflammatory drugs, niacin, oral hypoglycemics, pilocarpine, salicylates, or $\gamma$-butyrolactone.

\section{How Should Patients Bothered by Night Sweats Be Evaluated?}

Several approaches to the evaluation of patients reporting night sweats have been proposed. ${ }^{3,42,43}$ However, no published studies have evaluated the accuracy or cost-effectiveness of specific night sweat evaluation protocols. 
Table 4. Evidence Supporting Associations between Clinical Conditions and Elicited Subjective Night Sweats

\begin{tabular}{|c|c|c|c|c|}
\hline $\begin{array}{l}\text { Clinical } \\
\text { Disorder }\end{array}$ & & $\mathrm{N}$ & $\begin{array}{l}\text { Comparative } \\
\text { Data }\end{array}$ & $\begin{array}{c}\text { Proportion with Night Sweats (if Comparison } \\
\text { Group) or Proportion in Whom Night Sweats } \\
\text { Resolved with Treatment }\end{array}$ \\
\hline \multirow[t]{3}{*}{ Autoimmune } & POEMS syndrome & 1 & No & Disorder treated, night sweats resolved ${ }^{59}$ \\
\hline & Rheumatoid arthritis & 1 & No & Disorder treated, night sweats resolved ${ }^{60}$ \\
\hline & Giant cell arteritis & 1 & No & Disorder treated, night sweats resolved ${ }^{61}$ \\
\hline \multirow[t]{2}{*}{ Cardiovascular } & Aortic dissection, chronic & 1 & No & Disorder treated, night sweats resolved ${ }^{62}$ \\
\hline & Nocturnal/Prinzmetal angina & 1 & No & Disorder treated, night sweats persisted ${ }^{63}$ \\
\hline \multirow[t]{2}{*}{ Endocrine } & Diabetes insipidus & 1 & No & Disorder treated/night sweats resolved ${ }^{64}$ \\
\hline & Hyperthyroidism & 1 & No & Disorder treated, night sweats resolved ${ }^{65}$ \\
\hline GI & GERD & 12 & No & Disorder treated, night sweats resolved $(100 \%)^{57}$ \\
\hline \multirow{25}{*}{$\begin{array}{l}\text { Infectious } \\
\text { disease }\end{array}$} & HIV & & & \\
\hline & P24 Ag+, HIV- & 3816 & Yes & $5 / 58(9 \%)$ \\
\hline & P24 Ag-, HIV- & & & $38 / 3758(1 \%)^{66}$ \\
\hline & HIV-negative & 328 & Yes & $20 / 328(6 \%)$ night sweats \\
\hline & Acute HIV+ & & & $59 / 328(18 \%)$ \\
\hline & Chronic HIV+ & & & $39 / 328(12 \%)^{67}$ \\
\hline & HIV seroconverters & 110 & Yes & $18 / 22(82 \%)$ night sweats \\
\hline & Nonseroconverters & & & $6 / 88(7 \%)^{68}$ \\
\hline & $\begin{array}{l}\text { IV drug users, HIV+ } \\
\text { IV drug users, HIV- }\end{array}$ & 223 & Yes & $\begin{array}{l}33 / 124(27 \%) \text { night sweats } \\
13 / 99(13 \%)^{69}\end{array}$ \\
\hline & $\begin{array}{l}\text { Hodgkin disease, } \mathrm{HIV}+ \\
\text { Hodgkin disease, } \mathrm{HIV}-\end{array}$ & 16 & Yes & $\begin{array}{l}2 / 5(40 \%) \text { night sweats } \\
3 / 11(27 \%)^{70}\end{array}$ \\
\hline & $\begin{array}{l}\text { HIV+ with persistent } \\
\text { generalized } \\
\text { lymphadenopathy }\end{array}$ & 88 & Yes & $12 / 38(32 \%)$ night sweats \\
\hline & HIV + with lymphoma & & & $7 / 50(14 \%)^{71}$ \\
\hline & TB patients, $\mathrm{HIV}+$ & 158 & Yes & $24 / 48(50 \%)$ \\
\hline & TB patients, HIV- & & & $11 / 31(35 \%)^{72}$ \\
\hline & HIV patients, TB + & 899 & Yes & $\begin{array}{l}15 / 44(34 \%) \text { night sweats } \\
108 / 855(14 \%)^{73}\end{array}$ \\
\hline & HIV patients, TB- & 31 & No & $\begin{array}{l}\text { 27/31 (87\%) } 4.8 \text { episodes per week pretreatment; } 1.6 \\
\text { episodes/week posttreatment }{ }^{74}\end{array}$ \\
\hline & $\mathrm{HIV}+, \mathrm{MAC}$ & 25 & No & $\begin{array}{l}21 / 25(84 \%) \text { had night sweats pretreatment; } \\
\text { post treatment one of } 25(4 \%) \text { had night sweats }{ }^{75}\end{array}$ \\
\hline & & 24 & No & $\begin{array}{l}\text { 18/24 }(75 \%) \text { had night sweats pretreatment; } \\
\text { posttreatment } 6 / 24(25 \%) \text { had night sweats }{ }^{76}\end{array}$ \\
\hline & & 187 & No & $\begin{array}{l}67 / 187(36 \%) \text { had night sweats pretreatment; } \\
\text { posttreatment } 40 \% \text { had fewer night sweats when } \\
\text { treated with } 4 \text { drugs; }\end{array}$ \\
\hline & & 1748 & Yes & $70 \%$ had fewer when treated with 3 drugs $^{77}$ \\
\hline & $\mathrm{HIV}+, \mathrm{TB}+$ & & & 88/267 (33\%) had HIV+ with TB+ \\
\hline & $\mathrm{HIV}+, \mathrm{TB}-$ & & & 191/1471 (13\%) had HIV+ without TB \\
\hline & $\mathrm{HIV}+, \mathrm{TB}+$ & & & $128 / 267$ (48\%) had night sweats past 24 hours \\
\hline & $\mathrm{HIV}+, \mathrm{TB}-$ & & & $368 / 1471(25 \%)^{78}$ had night sweats past month \\
\hline & Viral URI/Mononucleosis & 356 & Yes & $\begin{array}{l}\text { Cohort matched on age, sex, date of diagnosis; night } \\
\text { sweats more common in mononucleosis }(P= \\
.000001)^{79}\end{array}$ \\
\hline
\end{tabular}

Continued 


\begin{tabular}{|c|c|c|c|c|}
\hline $\begin{array}{l}\text { Clinical } \\
\text { Disorder }\end{array}$ & & $\mathrm{N}$ & $\begin{array}{l}\text { Comparative } \\
\text { Data }\end{array}$ & $\begin{array}{c}\text { Proportion with Night Sweats (if Comparison } \\
\text { Group) or Proportion in Whom Night Sweats } \\
\text { Resolved with Treatment }\end{array}$ \\
\hline & \multicolumn{4}{|l|}{ Tuberculosis } \\
\hline & $\begin{array}{l}\text { Hospitalized patients awaiting } \\
\text { TB diagnosis ( } 44 \text { were TB- } \\
\text { positive) }\end{array}$ & 101 & Yes & $\begin{array}{l}\text { No significant difference in rate of night sweats } \\
\text { between those with TB and those without } \mathrm{TB}^{80}\end{array}$ \\
\hline & $\begin{array}{l}\text { Hospitalized patients in } \\
\text { isolation awaiting diagnosis }\end{array}$ & 563 & Yes & $\begin{array}{l}\text { 26/47 (55\%) of TB patients reported night sweats; } \\
\text { 141/516 (27\%) of non-TB patients reported night } \\
\text { sweats } 52,81\end{array}$ \\
\hline & $\begin{array}{l}\text { Emergency department patients } \\
\text { with sputum tested for TB }\end{array}$ & 145 & Yes & $\begin{array}{l}\text { 13/28 (46\%) with positive sputum reported night } \\
\text { sweats; } 29 / 113(26 \%) \text { with negative sputum } \\
\text { reported night sweats }{ }^{51}\end{array}$ \\
\hline & $\begin{array}{l}\text { Hospitalized patients awaiting } \\
\text { TB diagnosis }\end{array}$ & 101 & Yes & $\begin{array}{l}\text { 44/100 were TB-positive; no association between } \\
\text { reported night sweats and TB positivity }{ }^{80}\end{array}$ \\
\hline & Cysticercosis (Coenurus) & 1 & No & $\begin{array}{l}\text { Case history; night sweats and lymphoma-like } \\
\text { symptoms ceased with excision of larval cysts }\end{array}$ \\
\hline \multirow[t]{4}{*}{ Medications } & Antidepressants & & & \\
\hline & Sertraline & 1 & No & $\begin{array}{l}\text { Case history; night sweats resolved with } \\
\text { discontinuation of sertraline suggest night sweats } \\
\text { may be sign of "serotonin syndrome" } 83\end{array}$ \\
\hline & Venlafaxine & 1 & & $\begin{array}{l}\text { Case history; night sweats resolved when drug was } \\
\text { discontinued }^{84}\end{array}$ \\
\hline & Efavirenz & 1 & No & $\begin{array}{l}\text { Case history; night sweats resolved with } \\
\text { discontinuation of drug }\end{array}$ \\
\hline \multirow{9}{*}{$\begin{array}{l}\text { Neoplastic and } \\
\text { hematologic }\end{array}$} & Hemangioma, hepatic & 1 & No & Night sweats resolved when hemangioma removed ${ }^{86}$ \\
\hline & Lymphoma & 85 & No & $\begin{array}{l}\text { 4/14 }(29 \%) \text { with EBV Hodgkin disease had night } \\
\text { sweats; in } 75 \% \text {, night sweats improved after } \\
\text { treatment of disease }{ }^{87}\end{array}$ \\
\hline & Lymphadenopathy & 258 & Yes & 14/85 (17\%) patients with ТВ had night sweats \\
\hline & & & & $\begin{array}{l}\text { 24/98 (25\%) patients with lymphoma had night } \\
\text { sweats }\end{array}$ \\
\hline & & & & $\begin{array}{l}\text { 5/17 (29\%) patients with metastatic cancer had night } \\
\text { sweats }\end{array}$ \\
\hline & & & & $0 / 6(0 \%)$ of patients with sarcoidosis had night sweats \\
\hline & & & & $\begin{array}{l}5.36(14 \%) \text { of patients with nonspecific reactive } \\
\text { hyperplasia had night sweats } 88\end{array}$ \\
\hline & Myelofibrosis & 24 & No & $\begin{array}{l}21 / 24(88 \%) \text { night sweats improved }>50 \% \text { with } \\
\text { JAK1 and JAK2 inhibitor treatment }\end{array}$ \\
\hline & Prostate cancer, inflammatory & 1 & No & Case study; treated, night sweats resolved ${ }^{90}$ \\
\hline \multirow{5}{*}{$\begin{array}{l}\text { Psychiatric/ } \\
\text { behavioral }\end{array}$} & Depression & & & \\
\hline & $\mathrm{HIV}+$ and depressed & 62 & Yes & $16 / 28(57 \%)$ had night sweats \\
\hline & HIV+ and not depressed & & & $7 / 34(21 \%)^{91}$ \\
\hline & Night terrors & 1 & No & $\begin{array}{l}\text { Case study, night sweats resolved with treatment for } \\
\text { posttraumatic syndrome }{ }^{92}\end{array}$ \\
\hline & Panic disorder & 175 & No & $26 / 175(15 \%)$ of attacks occurred at night; \\
\hline \multirow{6}{*}{$\begin{array}{l}\text { Sleep } \\
\text { disturbance }\end{array}$} & & & & $46 / 175(26 \%)$ associated with sweating ${ }^{93}$ \\
\hline & Obstructive sleep apnea & & & \\
\hline & Snoring/daytime sleepiness & 4680 & Yes & 14/202 (14\%) with snoring/sleepiness; \\
\hline & No snoring/sleepiness & & & $179 / 4478(4 \%)$ with no snoring/sleepiness ${ }^{58}$ \\
\hline & Apnea-hypopnea index $\geq 15$ & 282 & Yes & Subjective night sweats in $28 / 114(25 \%)$ \\
\hline & Apnea-hypopnea index $<15$ & & & Subjective night sweats in 50/167 $(30 \%)^{38}$ \\
\hline
\end{tabular}

GI, gastrointestinal; GERD, gastroesophageal reflux disease; IV, intravenous; TB, tuberculosis; URI, upper respiratory infection; EBV, Epstein-Barr virus; MAC, Mycobacterium avium complex. 
Table 5. Prevalence of Subjective Night Sweats (Elicited) among Patients with Medical Conditions

\begin{tabular}{|c|c|}
\hline Condition & Prevalence in Specific Samples \\
\hline \multicolumn{2}{|l|}{ Autoimmune diseases } \\
\hline Polymorphic reticulosis & $7 / 34(21 \%)^{94}$ \\
\hline \multicolumn{2}{|l|}{ Endocrine diseases } \\
\hline Diabetes mellitus & $14 / 22(64 \%)$ with nocturnal hypoglycemia ${ }^{95}$ \\
\hline Obesity, severe in residential treatment of obesity & 20/152 (13\%) men; 47/234 (20\%) women ${ }^{96}$ \\
\hline \multicolumn{2}{|l|}{ Infectious diseases } \\
\hline Adenovirus, immunocompromised & $4 / 15(27 \%)^{97}$ \\
\hline Endocarditis, Cardiobacterium bominis & $14 / 61(24 \%)^{98}$ \\
\hline \multirow[t]{4}{*}{ HIV, acute } & $5 / 58(9 \%)$ of $\mathrm{p} 24 \mathrm{Ag}+/ \mathrm{HIV}-{ }^{66}$ \\
\hline & $7 / 20(35 \%)^{99}$ \\
\hline & $59 / 328(18 \%)^{67}$ \\
\hline & $88 / 151(58 \%)^{100}$ \\
\hline \multirow[t]{10}{*}{ HIV, chronic } & $18 / 22(82 \%)^{68}$ \\
\hline & $39 / 328(12 \%)^{67}$ \\
\hline & $19 / 49(31 \%)^{101}$ \\
\hline & $126 / 899(14 \%)^{73}$ \\
\hline & $7 / 34(21 \%)^{91}$ \\
\hline & $40 / 168(24 \%)^{102}$ \\
\hline & $26 / 100(26 \%)^{103}$ \\
\hline & $62 / 109(57 \%)^{104}$ \\
\hline & $144 / 205(70 \%)$ (average once/week) ${ }^{105}$ \\
\hline & 18/176 (10\%) with no AIDS-defining illnesses ${ }^{50}$ \\
\hline $\mathrm{HIV}+, \mathrm{IV}$ drug users & $33 / 124(27 \%)^{69}$ \\
\hline $\mathrm{HIV}+$, generalized lymphadenopathy & $12 / 38(32 \%)^{71}$ \\
\hline \multirow[t]{5}{*}{$\mathrm{HIV}+$, Mycobacterium avium complex } & $36 / 91(40 \%)^{106}$ \\
\hline & $27 / 31(87 \%)^{74}$ \\
\hline & $21 / 25(84 \%)^{75}$ \\
\hline & $18 / 24(75 \%)^{76}$ \\
\hline & $67 / 187(36 \%)^{77}$ \\
\hline $\mathrm{HIV}+$, Pneumocystis carinii pneumonia & $46 / 99(46 \%)^{107}$ \\
\hline $\mathrm{HIV}+$, pneumonia, bacterial & $22 / 94(23 \%)^{107}$ \\
\hline \multirow[t]{5}{*}{$\mathrm{HIV}+$, tuberculosis } & $66 / 85(78 \%)^{* 108}$ \\
\hline & $306 / 899(34 \%)^{73}$ \\
\hline & With pleural effusion $54 / 65(83 \%)^{109}$ \\
\hline & $19 / 36(53 \%)^{107}$ \\
\hline & $24 / 48(50 \%)^{72}$ \\
\hline Pneumonia, bacterial & $104 / 329(32 \%)^{110}$ \\
\hline \multirow[t]{10}{*}{ Tuberculosis } & $22 / 40(55 \%)^{111}$ \\
\hline & Young adults $179 / 419(43 \%)$, older adults $15 / 45(33 \%)^{112}$ \\
\hline & Young adults $34 / 73(46 \%)$, older adults $17 / 72(24 \%)^{113}$ \\
\hline & Young adults 48/88 (55\%), older adults $9 / 50(18 \%)^{114}$ \\
\hline & Young adults $14 / 29(48 \%)^{115}$ \\
\hline & Older adults $2 / 35(6 \%)^{115}$ \\
\hline & $12 / 17(71 \%)^{110}$ \\
\hline & $24 / 98(25 \%)$ TB patients presenting with lymphadenopathy ${ }^{88}$ \\
\hline & $148 / 313(48 \%)$ of TB outpatients ${ }^{116}$ \\
\hline & $11 / 31(35 \%)$ of TB outpatients ${ }^{72}$ \\
\hline \multirow[t]{2}{*}{ Tuberculosis, Beijing type } & Young adults $14 / 29(48 \%)$ \\
\hline & Older adults $2 / 35(6 \%)^{115}$ \\
\hline
\end{tabular}

Continued 
Table 5. Continued

\begin{tabular}{|c|c|}
\hline Condition & Prevalence in Specific Samples \\
\hline \multicolumn{2}{|l|}{ Tuberculosis } \\
\hline Beijing & $308 / 880(35 \%)$ \\
\hline Non-Beijing type & $378 / 880(43 \%)^{117}$ \\
\hline Beijing & $2 / 21(10 \%)$ \\
\hline Non-Beijing type & $7 / 20(35 \%)^{118}$ \\
\hline Tuberculosis, extrapulmonary & 99/209 (48\%) of patients with abdominal $\mathrm{TB}^{119}$ \\
\hline Medications & $28 / 54(54 \%)$ with extrapulmonary $\mathrm{TB}^{116}$ \\
\hline Oblimersen sodium & $7 / 40(18 \%)^{120}$ \\
\hline Tacatuzumab & $2 / 12(17 \%)^{121}$ \\
\hline \multicolumn{2}{|l|}{ Miscellaneous diseases } \\
\hline Inflammatory pseudotumor & $2 / 25(8 \%)^{122}$ \\
\hline \multicolumn{2}{|l|}{ Neoplastic and hematologic diseases } \\
\hline Acute lymphocytic leukemia & $8 / 10(80 \%)^{123}$ \\
\hline Acute myelocytic leukemia & $8 / 22(36 \%)^{123}$ \\
\hline \multirow[t]{5}{*}{ Lymphoma } & $14 / 85(17 \%)$ of those presenting with lymphadenopathy ${ }^{88}$ \\
\hline & $7 / 50(14 \%)$ with generalized lymphadenopathy ${ }^{71}$ \\
\hline & $3 / 10(30 \%)$ lymphoma primary in liver ${ }^{124}$ \\
\hline & 4/14 (29\%) with EBV Hodgkin disease ${ }^{87}$ \\
\hline & $5 / 12(42 \%)$ with pancreatic lymphoma ${ }^{87}$ \\
\hline Metastatic, mixed & $5 / 17(29 \%)$ of those presenting with lymphadenopathy ${ }^{88}$ \\
\hline Mesothelioma, peritoneal & $3 / 17(18 \%)^{125}$ \\
\hline \multirow[t]{2}{*}{ Mixed, terminal } & $12 / 77(16 \%)$ admission to hospice \\
\hline & $19 / 77(25 \%)$ just before death ${ }^{126}$ \\
\hline \multirow[t]{3}{*}{ Myelofibrosis } & $255 / 456(56 \%)^{127}$ \\
\hline & $8 / 22(36 \%)^{128}$ \\
\hline & $12 / 56(21 \%)^{129}$ \\
\hline Pancreatic cancer & $1 / 30(3 \%)^{130}$ \\
\hline Polycythemia vera & $198 / 405(49 \%)^{127}$ \\
\hline Prostate cancer, inflammatory & $7 / 12(58 \%)^{90}$ \\
\hline Thrombocythemia, essential & $125 / 304(41 \%)^{127}$ \\
\hline \multicolumn{2}{|l|}{ Pulmonary diseases } \\
\hline Eosinophilic pneumonia & $3 / 65(5 \%)^{131}$ \\
\hline \multicolumn{2}{|l|}{ Sleep disorders } \\
\hline Obstructive sleep apnea & $65 / 406(16 \%)^{132}$ \\
\hline \multicolumn{2}{|l|}{ Toxic and metabolic diseases } \\
\hline Silicone breast implants & $22 / 50(44 \%)^{133}$ \\
\hline
\end{tabular}

*Compilation of several case series.

EBV, Epstein-Barr virus; IV, intravenous; TB, tuberculosis.

\section{Aside from Treatment of the Underlying Cause, How Can Night Sweats Be Ameliorated?}

Few published studies have examined the question of symptomatic treatment of patients disturbed by frequent or severe night sweats, aside from the voluminous literature on postmenopausal night sweats and hot flushes. Those treatment approaches identified in our search are listed in Table 6.

Alpha adrenergic blockers may be effective in patients taking serotonin reuptake inhibitors. Nabilone, thalidomide, and thioridazine may be effective for patients with terminal cancer, and etanercept may work in patient with myelofibrosis. However, these studies were too small and underpowered to yield reliable conclusions.

\section{Is Nocturnal Sweating Associated with Adverse Health Outcomes?}

Two population-based analyses were conducted comparing mortality rates in patients with and without night sweats. ${ }^{46}$ Among one group of 842 primary care patients $\geq 65$ years of age, $24 \%$ died 
Table 6. Case Studies of Treatments Reported to Be Effective for Patients with Night Sweats

\begin{tabular}{|c|c|c|}
\hline Population & $\begin{array}{l}\text { Treatment (Route of Administration } \\
\text { Cited Only Where Provided) }\end{array}$ & Effectiveness \\
\hline $\begin{array}{l}\text { Patients with advanced cancer, referred } \\
\text { for palliative care }\end{array}$ & $\begin{array}{l}\text { Nabilone at } 1 \mathrm{mg} \text { at bedtime or } \\
\text { twice a day }\end{array}$ & $\begin{array}{l}4 / 4 \text { patients' night sweats improved (mean } 5.75 \\
\text { points on the ESAS) }\end{array}$ \\
\hline Patients with terminal cancer & Thalidomide at $100 \mathrm{mg}$ at bedtime & $\begin{array}{l}6 / 7 \text { patients improved an average of } 4 \text { points } \\
\text { on a } 5 \text {-point scale; recurred off of drug and } \\
\text { improved again with reinstitution of drug }\end{array}$ \\
\hline $\begin{array}{l}\text { Patients with advanced local or } \\
\text { metastatic cancer }\end{array}$ & $\begin{array}{l}\text { Thioridazine at } 10-30 \mathrm{mg} \text { at } \\
\text { bedtime nightly }\end{array}$ & $\begin{array}{l}\text { 15/17 patients reported improvement, poorly } \\
\text { quantified }^{136}\end{array}$ \\
\hline Patients with myelofibrosis & $\begin{array}{l}\text { Etanercept at } 25 \mathrm{mg} \text { subcutaneously } \\
\text { twice weekly for up to } 24 \text { weeks }\end{array}$ & $6 / 8(75 \%)$ improved with treatment ${ }^{128}$ \\
\hline $\begin{array}{l}\text { Patients treated for narcotic addiction } \\
\text { with methadone }\end{array}$ & Desloratadine at $5 \mathrm{mg}$ per day & $\begin{array}{l}2 / 2(100 \%) \text { reported relief from night sweats } \\
\text { within } 1 \text { day } 137\end{array}$ \\
\hline Patients taking SSRIs & Mirtazapine at $15-60 \mathrm{mg}$ per day & $\begin{array}{l}\text { One case: dose-dependent reduction in night } \\
\text { sweats }{ }^{138}\end{array}$ \\
\hline Patients taking SSRIs & Benztropine at $0.5 \mathrm{mg}$ at bedtime & One case: temporary relief from night sweats ${ }^{84}$ \\
\hline Patients taking SSRIs & $\begin{array}{l}\text { Terazosin at } 1 \mathrm{mg} \text { per day to } 2 \mathrm{mg} \\
\text { at bedtime }\end{array}$ & $\begin{array}{l}\text { Significant reduction in night sweats in } \\
2 / 2(100 \%) \text {, one after a day, the other after } 4 \\
\text { weeks }^{139}\end{array}$ \\
\hline Patients taking SSRIs & Terazosin & $\begin{array}{l}\text { Significant reduction in night sweats in } 20 / \\
20(100 \%)^{140}\end{array}$ \\
\hline Patients taking SSRIs & Clonidine at $0.1 \mathrm{mg}$ twice daily & $\begin{array}{l}\text { One case: significant relief of night sweats after } \\
3 \text { week }^{139}\end{array}$ \\
\hline
\end{tabular}

SSRIs, selective serotonin reuptake inhibitors; ESAS, Edmonton Symptom Assessment System (scored 0-10).

during the 8 years of follow-up. Night sweats persisted for $>1$ year in $50 \%$ of those who survived. No significant relationships were found between length of survival and history of night sweats in the month before enrollment after controlling for other predictors of mortality. The other analysis, described in the same journal article, involved 682 patients seen in a geriatrics clinic and followed for an average of 7 years. Again, there were no differences in survival between those who reported night sweats at time of the first visit and those who did not.

The occurrence of night sweats, as part of the so-called "B symptom complex," has been considered a predictor of poor outcomes in patients with Hodgkin lymphoma, ${ }^{47}$ yet when separated from the other B symptoms, weight loss and fever, the prognostic value of night sweats disappears. ${ }^{48,49}$ There is some evidence that in HIV-positive patients, the presence of night sweats is a weak predictor of earlier progression to AIDS. ${ }^{50}$ However, this may simply reflect the presence of an undiagnosed AIDS-related condition.

\section{Conclusions}

Published definitions of night sweats vary primarily by required level of symptom severity. Lack of standard definitions in literature hampers attempts to determine associations between night sweats and specific clinical conditions. We join the pleas of other clinicians for studies to include specific descriptors of how symptom-related information was obtained. Were symptoms volunteered, elicited, or were signs observed? Did descriptions include the duration, frequency, severity, and possibly the degree to which the symptom was bothersome to the patient or others? For night sweats and many other symptoms, it would be most helpful to know the incidence, prevalence, and duration in segments of the general population as well as those seen in clinical settings. There is a need to better understand stages in the natural history of a symptom: from onset to concern, to discussion with friends and family, to home-based remediation, to clinical presentation. Associations found in cross-sectional studies should be further examined in qualitative interviews, longitudinal cohorts, and considered in light of possible physiological mechanisms.

Mechanisms explaining patient-reported night sweats can be categorized as thermoregulatory, nonthermoregulatory, and increased awareness or vigilance. There is a lack of data on normal nocturnal sweating patterns for different age groups, sexes, and body locations. The accuracy of subjective reporting 
of sweating has not yet been validated, although it likely to be greater when sweating is more frequent or severe. For more meaningful studies, patients should be asked to report immediately after objective recording periods as to whether they noticed their usual amount of sweating at the time of observation.

Although many causes of night sweats have been suggested in the literature, few have been firmly established. Clinical wisdom suggesting that tuberculosis causes night sweats appears to be valid in younger adults, those with extrapulmonary disease, and those who also have HIV/AIDS. However, the 2 studies that attempted to develop clinical prediction rules for tuberculosis, and included night sweats as an initial predictor, did not include the symptom in their final prediction models. ${ }^{51,52}$ The combination of HIV/ AIDS and Mycobacterium avium complex appears most clearly to be associated with night sweats. The link between malignancies such as lymphoma and night sweats is less clear, and there is too little information available on which to base decisions about other medical conditions.

There has been little research on effectiveness of drugs to treat night sweats. Several small studies suggest that $\alpha$ adrenergic blocking agents may reduce night sweats in patients taking serotonin reuptake inhibitors, and the drugs thalidomide and thioridazine may benefit some terminal patients with cancer with night sweats. Anticholinergic agents are known to reduce sweating generally, and although no studies were found for this indication, these agents might be expected to reduce night sweats.

Most patients who report night sweats to their primary care clinicians probably do not have a serious disease causing the symptom. However, there is so little evidence regarding potential causes of night sweats that proposals or findings from any evaluation protocol must be viewed with extreme caution. Although it is reassuring that average life expectancy did not differ between those with night sweats and those without in 2 different studies of older primary care patients, very few of the patients in either study had night sweats that were severe.

This systematic review was conducted in search of published evidence to support the many assumptions of a symptom's clinical relevance. In the case of night sweats, we found very little evidence to support any sort of clinical recommendations. An additional intent of this article was to suggest a blueprint for reviewing and documenting research evidence about other symptoms. We have described the process and our findings to inform future research on night sweats and to promote similar searches about other symptoms for which too little is known.

\section{References}

1. Kroenke K. The interface between physical and psychological symptoms. Prim Care Companion J Clin Psychiatry 2003;5(suppl 7):11-8.

2. Ely JW, Osheroff JA, Ferguson KJ, Chambliss ML, Vinson DC, Moore JL. Lifelong self-directed learning using a computer database of clinical questions. J Fam Pract 1997;45:382-8.

3. Smetana GW, Aronson MD, Sokol HN. Approach to the patient with night sweats. Version 19.2. UpToDate. 2011. Available at: www.uptodate.com/ contents/approach-to-the-patient-with-night-sweats? source $=$ search_result\&search $=$ approach + to + the + patient + with + night + sweats\&selected Title $=1 \% 7$ E150. Accessed October 14, 2011.

4. Sato K, Kang WH, Saga K, Sato KT. Biology of sweat glands and their disorders. I. Normal sweat gland function. J Am Acad Dermatol 1989;20:537-63.

5. Sato K, Sato F. Individual variations in structure and function of human eccrine sweat gland. Am J Physiol 1983;245:R203-8.

6. Shibasaki M, Crandall CG. Mechanisms and controllers of eccrine sweating in humans. Front Biosci 2010;2:685-96.

7. Gobbi PG, Pieresca C, Ricciardi L, Vacchi S, Bertoloni D, Rossi A, Grignani G, Rutigliano L, Ascari E. Night sweats in Hodgkin's disease. A manifestation of preceding minor febrile pulses. Cancer 1990;65:2074-7.

8. Crandall CJ, Crawford SL, Gold EB. Vasomotor symptom prevalence is associated with polymorphisms in sex steroid-metabolizing enzymes and receptors. Am J Med 2006;119(suppl 1):S52-60.

9. Deecher DC, Dorries K. Understanding the pathophysiology of vasomotor symptoms (hot flushes and night sweats) that occur in perimenopause, menopause, and postmenopause life stages. Arch Womens Mental Health 2007;10:247-57.

10. IUPS Thermal Commission. Glossary of terms for thermal physiology. 3rd ed. Jpn J Physiol 2001;51:ixxxvi.

11. Tayefeh F, Plattner O, Sessler DI, Ikeda T, Marder D. Circadian changes in the sweating-to-vasoconstriction interthreshold range. Pflugers Archiv Eur J Physiol 1998;435:402-6.

12. Holtzclaw BJ. Circadian rhythmicity and homeostatic stability in thermoregulation. Biol Res Nurs 2001;2:221-35.

13. Timbal J, Colin J, Boutelier C. Circadian variations in the sweating mechanism. J Appl Physiol 1975; 39:226-30.

14. Morris C, Atkinson G, Drust B, Marrin K, Gregson $W$. Human core temperature responses during ex- 
ercise and subsequent recovery: an important interaction between diurnal variation and measurement site. Chronobiol Int 2009;26:560-75.

15. Aoki K, Kondo N, Shibasaki M, Takano S, Tominaga $H$, Katsuura T. Circadian variation of sweating responses to passive heat stress. Acta Physiol Scand 1997;161:397-402.

16. Kenny GP, Periard J, Journeay WS, Sigal RJ, Reardon FD. Effect of exercise intensity on the postexercise sweating threshold. J Appl Physiol 2003;95: 2355-60.

17. Kreider RB, Fry AC, O’Toole ML. Overtraining in sport. Champaign, IL: Human Kinetics; 1998. p. 5.

18. Armstrong LE, Stoppani J. Central nervous system control of heat acclimation adaptations: an emerging paradigm. Rev Neurosci 2002;13:271-85.

19. Abram WP, Allen JA, Roddie IC. The effect of pain on human sweating. J Physiol 1973;235:741-7.

20. Maple S, Bradshaw CM, Szabadi E. Pharmacological responsiveness of sweat glands in anxious patients and healthy volunteers. Br J Psychiatry 1982; 141:154-61.

21. van den Broek MD, Bradshaw CM, Szabadi E. The effects of a psychological 'stressor' and raised ambient temperature on the pharmacological responsiveness of human eccrine sweat glands: implications for sweat gland hyper-responsiveness in anxiety states. Eur J Clin Pharmacol 1984;26:209213.

22. Cabanac M. Adjustable set point: to honor Harold T. Hammel. J Appl Physiol 2006;100:1338-46.

23. Murphy PJ, Campbell SS. Nighttime drop in body temperature: a physiological trigger for sleep onset? Sleep 1997;20:505-11.

24. Avery D, Wildschiodtz G, Rafaelsen O. REM latency and temperature in affective disorder before and after treatment. Biol Psychiatry 1982;17: 463-70.

25. Avery DH. REM sleep and temperature regulation in affective disorder. In: Halaris A, editor. Chronobiology and neuropsychiatric disorders. New York, NY: Elsevier; 1987. p. 75-101.

26. Avery DH, Wildschiodtz G, Rafaelsen OJ. Nocturnal temperature in affective disorder. J Affect Disord $1982 ; 4: 61-71$.

27. Avery DH, Wildschiodtz G, Smallwood RG, Martin D, Rafaelsen OJ. REM latency and core temperature relationships in primary depression. Acta Psychiatr Scand 1986;74:269-80.

28. Schwartz PJ, Rosenthal NE, Kajimura N, Han L, Turner EH, Bender C, Wehr TA. Ultradian oscillations in cranial thermoregulation and electroencephalographic slow-wave activity during sleep are abnormal in humans with annual winter depression. Brain Res 2000;866:152-67.
29. Szuba MP, Guze BH, Baxter LR Jr. Electroconvulsive therapy increases circadian amplitude and lowers core body temperature in depressed subjects. Biol Psychiatry 1997;42:1130-7.

30. von Zerssen D, Barthelmes H, Dirlich G, Doerr P, Emrich HM, von Lindern L, Lund R, Pirke KM. Circadian rhythms in endogenous depression. Psychiatry Res 1985;16:51-63.

31. Avery DH, Shah SH, Eder DN, Wildschiodtz G. Nocturnal sweating and temperature in depression. Acta Psychiatr Scand 1999;100:295-301.

32. Gnirss F, Schneider D. Patterns of spontaneous autonomic activities during human NREM sleep. In: Levin P, Koella WP, editors. Sleep. Basel, Switzerland: Karger; 1975. p. 352-5.

33. Ogawa T, Low PA. Autonomic regulation of temperature and sweating. In: Low PA, editor. Clinical autonomic disorders. Philadelphia, PA: LippincottRaven Publishers; 1997, p. 83-96.

34. Noll G, Elam M, Kunimoto M, Karlsson T, Wallin BG. Skin sympathetic nerve activity and effector function during sleep in humans. Acta Physiol Scand 1994;151:319-29.

35. Spreng LF, Johnson LC, Lubin A. Autonomic correlates of eye movement bursts during stage REM sleep. Psychophysiology 1968;4:311-23.

36. Wehr TA. A brain-warming function for REM sleep. Neurosci Biobehav Rev 1992;16:379-97.

37. Mold JW, Mathew MK, Belgore S, DeHaven M. Prevalence of night sweats in primary care patients: an OKPRN and TAFP-Net collaborative study. J Fam Pract 2002;51:452-6.

38. Mold JW, Goodrich S, Orr W. Associations between subjective night sweats and sleep study findings. JABFM 2008;21:96-100.

39. Mold JW, Woolley JH, Nagykaldi Z. Associations between night sweats and other sleep disturbances: an OKPRN study. Ann Fam Med 2006;4:423-6.

40. Mold JW, Roberts M, Aboshady HM. Prevalence and predictors of night sweats, day sweats, and hot flashes in older primary care patients: an OKPRN study. Ann Fam Med 2004;2:391-7.

41. Cheong YS, Mold JW, Park EW. The epidemiology of night sweats in a South Korean primary care population: a Chunan Network study. 2002. Unpublished.

42. Viera AJ, Bond MM, Yates SW. Diagnosing night sweats. Am Family Phys 2003;67:1019-24.

43. Su CW, Gaskie S, Hitchcock K, Johnson L, Su CW, Gaskie S, Hitchcock K, Johnson L. Clinical inquiries. What's the best diagnostic evaluation of night sweats? J Family Pract 2007;56:493-5.

44. Chambliss ML. Frequently asked questions from clinical practice. What is the appropriate diagnostic approach for patients who complain of night sweats? Arch Fam Med 1999;8:168-9.

45. Dall L, Stanford JF. Fever, chills, and night sweats. In: Walker HK, Hall WD, Hurst JW, editors. Clin- 
ical methods: the history, physical, and laboratory examinations. 3rd ed. Boston, MA, London, UK: Butterworths; 1990. p. 944-8.

46. Mold JW, Lawler F. The prognostic implications of night sweats in two cohorts of older patients. J Am Board Fam Med 2010;23:97-103.

47. Lister TA, Crowther D, Sutcliffe SB, Glatstein E, Canellos GP, Young RC, Rosenberg SA, Coltman CA, Tubiana M. Report of a committee convened to discuss the evaluation and staging of patients with Hodgkin's disease: Cotswolds meeting. J Clin Oncol 1989;7:1630-6.

48. Gobbi PG, Cavalli C, Gendarini A, Crema A, Ricevuti G, Federico M, Di Prisco U, Ascari E. Reevaluation of prognostic significance of symptoms in Hodgkin's disease. Cancer 1985;56:2874-80.

49. Crnkovich MJ. Stage I to IIB Hodgkin's disease: the combined experience at Stanford University and the Joint Center for Radiation Therapy. J Clin Oncol 1987;5:1041.

50. Rabeneck L, Crane MM, Risser JM, Lacke CE, Wray NP. A simple clinical staging system that predicts progression to AIDS using CD4 count, oral thrush, and night sweats. J Gen Intern Med 1993;8:5-9.

51. Redd JT, Susser E. Controlling tuberculosis in an urban emergency department: a rapid decision instrument for patient isolation. Am J Public Health 1997;87:1543-7.

52. El-Solh A, Mylotte J, Sherif S, Serghani J, Grant BJ. Validity of a decision tree for predicting active pulmonary tuberculosis. Am J Respir Crit Care Med 1997;155:1711-6.

53. Smetana GW. Diagnosis of night sweats. JAMA 1993;270:2502-3.

54. Holtzclaw BJ. Fever and night sweats. In: Casey K, Cohen F, Halloran J, Hughes A, editors. ANAC core curriculum for HIV/AIDS nursing. Malibu, CA: NurseCom; 1996. p. 211-7.

55. Lea MJ, Aber RC. Descriptive epidemiology of night sweats upon admission to a university hospital. South Med J 1985;78:1065-67.

56. Quigley CS, Baines M. Descriptive epidemiology of sweating in a hospice population. J Palliat Care 1997;13:22-6.

57. Reynolds WA. Are night sweats a sign of esophageal reflux? J Clin Gastroenterol 1989;11:590-1.

58. Suwanprathes P, Won C, Komoltri C, Nana A, Kotchabhakdi N, Guilleminault C. Epidemiology of sleep-related complaints associated with sleepdisordered breathing in Bangkok, Thailand. Sleep Med 2010;11:1025-30.

59. Hudnall SD, Chen T, Brown K, Angel T, Schwartz MR, Tyring SK. Human herpesvirus-8-positive microvenular hemangioma in POEMS syndrome. Arch Pathol Lab Med 2003;127:1034-6.

60. Crouch R, Akhras V, Sarkany R. Schnitzler's syn- drome: successful treatment with anakinra. Aust J Dermatol 2007;48:178-81.

61. Morris GC, Thomas TP. Night sweats-presentation of an often forgotten diagnosis. Br J Clin Pract $1991 ; 45: 145$.

62. Schattner A, Klepfish A, Caspi A. Chronic aortic dissection presenting as a prolonged febrile disease and arterial embolization. Chest 1996;110:1111-4.

63. Becker RC, Giuliani M, Alpert JS. Nocturnal diaphoresis and coronary artery spasm. Contribution of the parasympathetic nervous system. Cleve Clin J Med 1987;54:515-8.

64. Raff SB, Gershberg H. Night sweats. A dominant symptom in diabetes insipidus. JAMA 1975;234: 1252-3.

65. Jayapaul M, Williams MR, Davies DP, Large DM. Recurrent painful unilateral gynaecomastia-interactions between hyperthyroidism and hypogonadism. Andrologia 2006;38:31-3.

66. Bollinger RC, Brookmeyer RS, Mehendale SM, Paranjape RS, Shepherd ME, Gadkari DA, Quinn TC. Risk factors and clinical presentation of acute primary HIV infection in India. JAMA 1997;278: 2085-9.

67. Veugelers PJ, Kaldor JM, Strathdee SA, Page-Shafer KA, Schechter MT, Coutinho RA, Keet IP, van Griensven GJ. Incidence and prognostic significance of symptomatic primary human immunodeficiency virus type 1 infection in homosexual men. J Infect Dis 1997;176:112-7.

68. Fox R, Eldred LJ, Fuchs EJ, Kaslow RA, Visscher BR, Ho M, Phair JP, Polk BF. Clinical manifestations of acute infection with human immunodeficiency virus in a cohort of gay men. AIDS 1987;1: $35-8$.

69. El-Sadr W, Goetz RR, Sorrell S, Joseph M, Ehrhardt A, Gorman JM. Clinical and laboratory correlates of human immunodeficiency virus infection in a cohort of intravenous drug users from New York, NY. Arch Intern Med 1992;152:1653-9.

70. Poluri A, Shah KG, Carew JF, Shaha AR, Har-El G, Lucente FE, Singh B. Hodgkin's disease of the head and neck in human immunodeficiency virusinfected patients. Am J Otolaryngol 2002;23:12-6.

71. Tirelli U, Vaccher E, Zagonel V, Saracchini S, Bertola G, Serraino D, Monfardini S, Carbone A. Persistent generalized lymphadenopathy syndrome vs 'AIDS'-unrelated malignant lymphoma: comparison of presenting clinical and laboratory findings in 88 patients. AIDS and Related Syndromes Study Group. Tumori 1989;75:222-5.

72. Corbett EL, Zezai A, Cheung YB, Bandason T, Dauya E, Munyati SS, Butterworth AE, Rusikaniko S, Churchyard GJ, Mungofa S, Hayes RJ, Mason PR. Provider-initiated symptom screening for tuberculosis in Zimbabwe: diagnostic value and the effect of HIV status. Bull World Health Organ 2010;88:13-21. 
73. Day JH, Charalambous S, Fielding KL, Hayes RJ, Churchyard GJ, Grant AD. Screening for tuberculosis prior to isoniazid preventive therapy among $\mathrm{HIV}$-infected gold miners in South Africa. Int J Tuberc Lung Dis 2006;10:523-9.

74. Kemper CA, Meng TC, Nussbaum J, Chiu J, Feigal DF, Bartok AE, Leedom JM, Tilles JG, Deresinski SC, McCutchan JA. Treatment of Mycobacterium avium complex bacteremia in AIDS with a fourdrug oral regimen. Rifampin, ethambutol, clofazimine, and ciprofloxacin. The California Collaborative Treatment Group. Ann Intern Med 1992;116: 466-72.

75. Hoy J, Mijch A, Sandland M, Grayson L, Lucas R, Dwyer B. Quadruple-drug therapy for Mycobacterium avium-intracellulare bacteremia in AIDS patients. J Infect Dis 1990;161:801-5.

76. Young LS, Wiviott L, Wu M, Kolonoski P, Bolan $\mathrm{R}$, Inderlied CB. Azithromycin for treatment of Mycobacterium avium-intracellulare complex infection in patients with AIDS. Lancet 1991;338: 1107-9.

77. Singer J, Thorne A, Khorasheh S, Raboud JM, Wu AW, Salit I, Tsoukas CM, Lemieux C, Shafran SD. Symptomatic and health status outcomes in the Canadian randomized MAC treatment trial (CTN010). Canadian HIV Trials Network Protocol 010 Study Group. Int J STD AIDS 2000;11:212-9.

78. Cain KP, McCarthy KD, Heilig CM, Monkongdee P, Tasaneeyapan T, Kanara N, Kimerling ME, Chheng P, Thai S, Sar B, Phanuphak P, Teeratakulpisarn N, Phanuphak N, Nguyen HD, Hoang TQ, Le HT, Varma JK. An algorithm for tuberculosis screening and diagnosis in people with HIV. N Engl J Med 2010;362:707-16.

79. Lambore S, McSherry J, Kraus AS. Acute and chronic symptoms of mononucleosis. J Family Pract 1991;33:33-7.

80. Cohen R, Muzaffar S, Capellan J, Azar H, Chinikamwala $M$. The validity of classic symptoms and chest radiographic configuration in predicting pulmonary tuberculosis. Chest 1996;109:420-3.

81. El-Solh AA, Hsiao CB, Goodnough S, Serghani J, Grant BJ. Predicting active pulmonary tuberculosis using an artificial neural network. Chest 1999;116: $968-73$

82. Benger A, Rennie RP, Roberts JT, Thornley JH, Scholten T. A human coenurus infection in Canada. Am J Trop Med Hyg 1981;30:638-44.

83. Babbott SF, Pearson VE. Sertraline-related night sweats. Ann Intern Med 1999;130:242-3.

84. Pierre JM, Guze BH. Benztropine for venlafaxineinduced night sweats. J Clin Psychopharmacol 2000;20:269.

85. Martin AF, Figueroa SC, Merino Mde L, Hurlee AD. Hyperhidrosis in association with efavirenz. AIDS Patient Care STDS 2009;23:143-5.

86. Deutsch GS, Yeh KA, Bates WB, Tannehill WB.
Embolization for management of hepatic hemangiomas. Am Surg 2001;67:159-64.

87. Bollard CM, Aguilar L, Straathof KC, Gahn B, Huls MH, Rousseau A, Sixbey J, Gresik MV, Carrum G, Hudson M, Dilloo D, Gee A, Brenner MK, Rooney CM, Heslop HE. Cytotoxic T lymphocyte therapy for Epstein-Barr virus ${ }^{+}$Hodgkin's disease. J Exp Med 2004;200:1623-33.

88. Abba AA, Bamgboye AE, Afzal M, Rahmatullah RA. Lymphadenopathy in adults. A clinicopathological analysis. Saudi Med J 2002;23:282-6.

89. Pardanani A, Gotlib JR, Jamieson C, Cortes JE, Talpaz M, Stone RM, Silverman MH, Gilliland DG, Shorr J, Tefferi A. Safety and efficacy of TG101348, a selective JAK2 inhibitor, in myelofibrosis. J Clin Oncol 2011;29:789-96.

90. Mauri D, Pentheroudakis G, Tolis C, Chojnacka M, Pavlidis N. Inflammatory prostate cancer: an underestimated paraneoplastic clinical manifestation. Urol Oncol 2005;23:318-22.

91. Kalichman SC, Sikkema KJ, Somlai A. Assessing persons with human immunodeficiency virus (HIV) infection using the Beck Depression Inventory: disease processes and other potential confounds. J Pers Assess 1995;64:86-100.

92. Marshall JR. The treatment of night terrors associated with the posttraumatic syndrome. Am J Psychiatry 1975;132:293-5.

93. Margraf J, Taylor B, Ehlers A, Roth WT, Agras WS. Panic attacks in the natural environment. J Nerv Ment Dis 1987;175:558-65.

94. Smalley SR, Cupps RE, Anderson JA, Ilstrup DM, McDonald TJ, Weiland LH, Deremee RD. Polymorphic reticulosis limited to the upper aerodigestive tract-natural history and radiotherapeutic considerations. Int J Radiat Oncol Biol Phys 1988; 15:599-605.

95. Gale EA, Tattersall RB. Unrecognised nocturnal hypoglycaemia in insulin-treated diabetics. Lancet 1979;1:1049-52.

96. Wachholtz A, Binks M, Suzuki A, Eisenson H. Sleep disturbance and pain in an obese residential treatment-seeking population. Clin J Pain 2009;25: 584-9.

97. Zahradnik JM, Spencer MJ, Porter DD. Adenovirus infection in the immunocompromised patient. Am J Med 1980;68:725-32.

98. Malani AN, Aronoff DM, Bradley SF, Kauffman CA. Cardiobacterium hominis endocarditis: two cases and a review of the literature. Eur J Clin Microbiol Infect Dis 2006;25:587-95.

99. Gaines H, von Sydow M, Pehrson PO, Lundbegh P. Clinical picture of primary HIV infection presenting as a glandular-fever-like illness. BMJ 1988; 297:1363-8.

100. Deeks SG, Kitchen CMR, Liu L, Guo H, Gascon R, Narvaez AB, Hunt P, Martin JN, Kahn JO, Levy J, McGrath MS, Hecht FM. Immune activation set 
point during early HIV infection predicts subsequent CD4+ T-cell changes independent of viral load. Blood 2004;104:942-7.

101. Yoong KY, Cheong I. A study of Malaysian drug addicts with human immunodeficiency virus infection. Int J STD AIDS 1997;8:118-23.

102. Kremer H, Sonnenberg-Schwan U, Arendt G, Brockmeyer NH, Potthoff A, Ulmer A, Graefe K, Lorenzen T, Starke W, Walker UA. HIV or HIVtherapy? Causal attributions of symptoms and their impact on treatment decisions among women and men with HIV. Eur J Med Res 2009;14:139-46.

103. Ajuluchukwu DC, Brown LS Jr, Crummey FC, Foster KF Sr, Ismail YI, Siddiqui N. Demographic, medical history and sexual correlates of HIV seropositive methadone maintained women. J Addict Dis 1993;12:105-20.

104. Johnson MO, Stallworth T, Neilands TB. The drugs or the disease? Causal attributions of symptoms held by HIV-positive adults on HAART. AIDS Behav 2003;7:109-17.

105. Cunningham WE, Shapiro MF, Hays RD, Dixon WJ, Visscher BR, George WL, Ettl MK, Beck CK. Constitutional symptoms and health-related quality of life in patients with symptomatic HIV disease. Am J Med 1998;104:129-36.

106. Singh VR, Smith DK, Lawerence J, Kelly PC, Thomas AR, Spitz B, Sarosi GA. Coccidioidomycosis in patients infected with human immunodeficiency virus: review of 91 cases at a single institution. Clin Infect Dis 1996;23:563-8.

107. Selwyn PA, Pumerantz AS, Durante A, Alcabes PG, Gourevitch MN, Boiselle PM, Elmore JG. Clinical predictors of Pneumocystis carinii pneumonia, bacterial pneumonia and tuberculosis in $\mathrm{HIV}$-infected patients. AIDS 1998;12:885-93.

108. Benson CA. Disease due to the Mycobacterium avium complex in patients with AIDS: epidemiology and clinical syndrome. Clin Infect Dis 1994; 3(suppl):S218-22.

109. Richter C, Perenboom R, Mtoni I, Kitinya J, Chande H, Swai AB, Kazema RR, Chuwa LM. Clinical features of HIV-seropositive and HIV-seronegative patients with tuberculous pleural effusion in Dar es Salaam, Tanzania. Chest 1994;106: 1471-5.

110. Liam C-K, Pang Y-K, Poosparajah S. Pulmonary tuberculosis presenting as community-acquired pneumonia. Respirology 2006;11:786-92.

111. Korzeniewska-Kosela M, FitzGerald JM, Vedal S, Allen EA, Schechter MT, Lawson L, Phillips P, Black W, Montaner JS. Spectrum of tuberculosis in patients with HIV infection in British Columbia: report of 40 cases. CMAJ 1992;146:1927-34.

112. Cavalcanti ZdR, de Albuquerque MdFPM, Campello AR, Ximenes R, Montarroyos U, Vercosa MK. Characteristics of elderly tuberculosis patients in Recife,
Brazil: a contribution to the tuberculosis control program. J Bras Pneumol 2006;32:535-43.

113. Van den Brande P, Vijgen J, Demedts M. Clinical spectrum of pulmonary tuberculosis in older patients: comparison with younger patients. J Gerontol 1991;46:M204-9.

114. Rawat J, Sindhwani G, Juyal R. Clinico-radiological profile of new smear positive pulmonary tuberculosis cases among young adult and elderly people in a tertiary care hospital at Deheradun (Uttarakhand). Indian J Tuberc 2008;55:84-90.

115. Alvarez S, Shell C, Berk SL. Pulmonary tuberculosis in elderly men. Am J Med 1987;82:602-6.

116. Miller LG, Asch SM, Yu EI, Knowles L, Gelberg L, Davidson P. A population-based survey of tuberculosis symptoms: how atypical are atypical presentations? Clin Infect Dis 2000;30:293-9.

117. Drobniewski F, Balabanova Y, Nikolayevsky V, Ruddy M, Kuznetzov S, Zakharova S, Melentyev A, Fedorin I. Drug-resistant tuberculosis, clinical virulence, and the dominance of the Beijing strain family in Russia. JAMA 2005;293:2726-31.

118. Sun YJ, Lim TK, Ong AK, Ho BC, Seah GT, Paton NI. Tuberculosis associated with Mycobacterium tuberculosis Beijing and non-Beijing genotypes: a clinical and immunological comparison. BMC Infect Dis 2006;6:105.

119. Khan R, Abid S, Jafri W, Abbas Z, Hameed K, Ahmad Z. Diagnostic dilemma of abdominal tuberculosis in non-HIV patients: an ongoing challenge for physicians. World J Gastroenterol 2006;12: 6371-5.

120. O'Brien SM, Cunningham CC, Golenkov AK, Turkina AG, Novick SC, Rai KR. Phase I to II multicenter study of oblimersen sodium, a Bcl-2 antisense oligonucleotide, in patients with advanced chronic lymphocytic leukemia. J Clin Oncol 2005; 23:7697-702.

121. Furman RR, Forero-Torres A, Shustov A, Drachman JG. A phase I study of dacetuzumab (SGN-40, a humanized anti-CD40 monoclonal antibody) in patients with chronic lymphocytic leukemia. Leuk Lymphoma 2010;51:228-35.

122. Moran CA, Suster S, Abbondanzo SL. Inflammatory pseudotumor of lymph nodes: a study of 25 cases with emphasis on morphological heterogeneity. Hum Pathol 1997;28:332-8.

123. Adedeji MO. The acute leukaemias in adults in Benin City, Nigeria. East Afr Med J 1989;66:64-8.

124. Osborne BM, Butler JJ, Guarda LA. Primary lymphoma of the liver. Ten cases and a review of the literature. Cancer 1985;56:2902-10.

125. Le DT, Deavers M, Hunt K, Malpica A, Verschraegen CF. Cisplatin and irinotecan (CPT-11) for peritoneal mesothelioma. Cancer Invest 2003; 21:682-9.

126. Tsai J-S, Wu C-H, Chiu T-Y, Hu W-Y, Chen C-Y. Symptom patterns of advanced cancer pa- 
tients in a palliative care unit. Palliat Med 2006;20: 617-22.

127. Mesa RA, Niblack J, Wadleigh M, Verstovsek S, Camoriano J, Barnes S, Tan $\mathrm{AD}$, Atherton PJ, Sloan JA, Tefferi A. The burden of fatigue and quality of life in myeloproliferative disorders (MPDs): an international Internet-based survey of 1179 MPD patients. Cancer 2006;109:68-76.

128. Steensma DP, Mesa RA, Li C-Y, Gray L, Tefferi A. Etanercept, a soluble tumor necrosis factor receptor, palliates constitutional symptoms in patients with myelofibrosis with myeloid metaplasia: results of a pilot study. Blood 2002;99:2252-4.

129. Varki A, Lottenberg R, Griffith R, Reinhard E. The syndrome of idiopathic myelofibrosis. A clinicopathologic review with emphasis on the prognostic variables predicting survival. Medicine 1983; 62:353-71.

130. Qiu L, Luo Y, Peng Y-L. Value of ultrasound examination in differential diagnosis of pancreatic lymphoma and pancreatic cancer. World J Gastroenterol 2008; 14:6738-42.

131. Capewell S, Chapman BJ, Alexander F, Greening AP, Crompton GK. Pulmonary eosinophilia with systemic features: therapy and prognosis. Respir Med 1992;86:485-90.

132. Wahner-Roedler DL, Olson EJ, Narayanan S, Sood R, Hanson AC, Loehrer LL, Sood A. Gender-specific differences in a patient population with obstructive sleep apnea-hypopnea syndrome. Gender Med 2007;4:329-38.

133. Freundlich B, Altman C, Snadorfi N, Greenberg M, Tomaszewski J. A profile of symptomatic patients with silicone breast implants: a Sjogrens-like syndrome. Semin Arthritis Rheum 1994;24(suppl 1):44-53.

134. Maida V. Nabilone for the treatment of paraneoplastic night sweats: a report of four cases. J Palliat Med 2008;11:929-34.

135. Deaner PB. The use of thalidomide in the management of severe sweating in patients with advanced malignancy: trial report. Palliat Med 2000;14:429-31.

136. Regnard C. Use of low-dose thioridazine to control sweating in advanced cancer. Palliat Med 1996;10: $78-9$.

137. Al-Adwani A, Basu N. Methadone and excessive sweating. Addiction 2004;99:259.

138. Buecking A, Vandeleur CL, Khazaal Y, Zullino DF. Mirtazapine in drug-induced excessive sweating. Eur J Clin Pharmacol 2005;61:543-4.

139. Mago R, Monti D. Antiadrenergic treatment of antidepressant-induced excessive sweating in 3 patients. J Clin Psychiatry 2007;68:639-40.

140. Mago R. Antidepressant-induced excessive sweating (ADIES): measurement and treatment. Presented at American Psychiatric Association; 2009; Philadelphia, PA. 